ISSN 1991- 8690

website : http:// jsci.utq.edu.iq

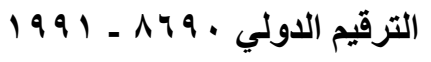

Email: utjsci@utq.edu.iq

\title{
Detection of malignant Cases by Segmentation of Cells in Medical Images and Applying Fuzzy Logic Technique
}

\author{
Salim J. Attia ${ }^{1}$ \\ Ziad M. Abood ${ }^{2}$ \\ Ibrahim R. Agool ${ }^{3}$ \\ ${ }_{2}^{1}$ College of Dentistry- Baghdad University \\ ${ }^{2}$ College of Education- Almustasiriyah University \\ ${ }^{3}$ College of Science - Almustasiriyah University
}

\begin{abstract}
$\underline{\text { Abstract }}$
The process of detection and segmentation of cells is considered in digital optical images of human breast tissue as important base to diagnose the diseases. The major features of malignancy are related with the cells. It is therefore essential to operate a segmentation of the image, to isolate the cells from the rest of image, i. e., from other tissue components, and from some other undesirable elements of images. The recognition process includes a segmentation algorithm based on an adaptive imaging threshold procedure that is sensitive to local ranges in pixel intensity (minimum-maximum values). The statistical features are extracted from the images of cells like median, mode, mean and standard deviation. Then the fuzzy logic method is applied to detect breast cancer.
\end{abstract}

Keys words: segmentation, adaptive threshold, optical imaging, fuzzy logic.

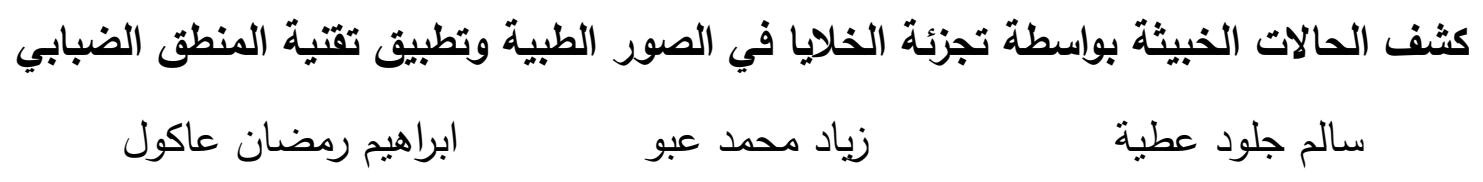

الخلاصة

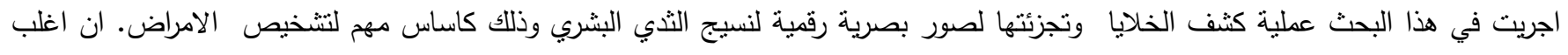

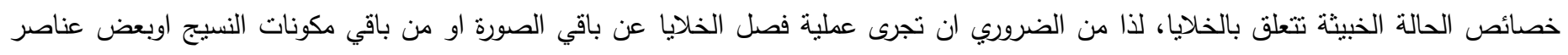

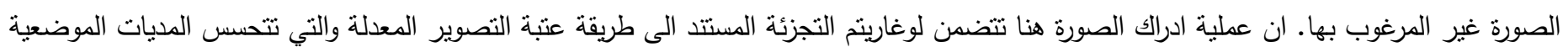

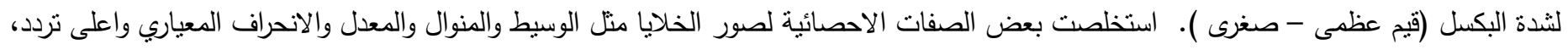

$$
\text { ثم طبق المنطق الضبابي لكثف سرطان الثدي. }
$$

\section{Introduction}

Image segmentation is the process of dividing an image into meaningful regions. The ideal case is to have just two regions of interest: the object region and the background region ${ }^{(1)}$. The design of image segmentation algorithms is a broad and active field, not only in medical imaging, but in a wide range of computer vision systems for nondestructive evaluation and satellite imagery, for example $\left.{ }^{(}\right)$. Its purpose is to divide an image into regions which are meaningful in a computational sense (e.g. for generating a feature vector) appropriate for a particular task or set of tasks ${ }^{(r)}$ . A wide variety of methodologies and approaches are used, the exact choice of any particular methodology depending upon the characteristics of the problem to be solved and its place in a wider image analysis strategy. 
Segmentation is an essential step prior to the description, recognition or classification of an image or its constituents. There are two major approaches: region-based methods, in which similarities are detected, and boundary-based methods, which rely on detecting the edges associated with an object, thereby defining a fundamental feature of the object $(\varepsilon)$. Several segmentation methods have been proposed in the literature, such as edge detection or hierarchic threshold or two dimensional (2D) histogram threshold (4). Locally the method of by determination of cell used most area of nuclei by segment square region in the nuclei (5).

\section{Fuzzy logic}

The idea of fuzzy logic proposed by Lotfi Zadeh (6) suggested that set membership is the key to decision making when faced with uncertainty. This notion of set membership, then, is central to the representation of objects within a universe by sets defined on the universe. Classical sets contain objects that satisfy precise properties of membership; fuzzy sets contain objects that satisfy imprecise properties of membership, i.e., membership of an object in a fuzzy set can be approximate. For example, the set of heights from 5 to 7 feet is precise (crisp); the set of heights in the region around 6 feet is imprecise, or fuzzy. So the membership function of classical set gives full membership or not (1 or 0 ), but the membership function of fuzzy set gives ratio of membership $(20 \%, 80 \% \ldots)$..(7) In classical set the membership function gives only two values. For any set A defined on the universe $\mathrm{X}$, there exists a functiontheoretic set, called a value set under the mapping of the characteristic function, $\chi$. By convention, the null set is assigned the membership value 0 and the whole set is assigned the membership value 1. (8) A fuzzy set, then, is a set containing elements that have varying degrees of membership in the set. This idea is in contrast with classical, or crisp, sets because members of a crisp set would not be members unless their membership was full, or complete, in that set (i.e., their membership is assigned a value of 1). Elements in a fuzzy set, because their membership need not be complete, can also be members of other fuzzy sets on the same universe.(8)

\section{Materials and Methods}

Samples of slides contain different normal and abnormal breast tissues taken by biopsy method are treated with $(\mathrm{H} \& \mathrm{E})$ stain and imaged by microscope with digital camera connected to computer as a system prepared for this goal, then applying the segmentation technique called adaptive imaging threshold procedure to separate the cells.(9-10)

\section{Result and discussion}

The segmentation is used to separate the regions of interest in obtained images from background surrounded them. In this study the cells in images are determined using the program which is its algorithm was explained below (segmentation algorithm):

First step: read image
Input image
Second step: threshold image
Move the mouse across the region of interest or
subimage i(x,y)
and determine which color is more affected by varying
of max-min values
$\quad$ Ave min $<$ Ave < Ave max
Record the range values (min, max) for region of
interest (ROI) in Image for R, G and B bands
Third step: segmentation of image
Do the segmentation process of the regions which have
those values at (min-max) threshold
Fourth step: check resulted image
If the results is good
save the output image

The segmentation of image aimed for recognize the objects of interest, i. e., to find suitable base that allow us to distinguish them from other objects and from the background. This step is to check each individual pixel to see whether it belongs to an object of interest or not.The color of the images is exploited, starting by the segmentation of cells based on computing the average value of the basic (R; G; B) color bands for the intercellular regions. The maximum and minimum limited values of these regions are computed for each color.We determine the average value using equation (11) 
Gray value average $=\frac{(R+G+B)}{3}$

Then we use this value for all color components according to suitable condition with equation: (11)

$$
\mathrm{A} v e_{\min }<\mathrm{A} v e<\mathrm{A} v e_{\max }
$$

The result provides a primary segmented image as see in Figure (1) which shows the original image and segmented cells for benign case. The same approach of segmentation of images is applied here to separate the malignant cells from original images. Determination of maximum and minimum limited values of the regions of interest are computed for each color by moving the pointer in boundaries of some cells to try and know the suitable values which give best segmentation of cells. We determine the average value using the equation (1) and inserting the value for all color components according to condition suitable with equation (2), as see in Figure (1) which shows the original image and segmented cells of malignant case.In the current application, we exploit the color of the images, starting by considering the segmentation of cells based on computing the average value of the basic $(R, G, B)$ color component for the intercellular zones. The 'pointer' is moved on these zones to obtain the maximum max and minimum min limited values for each color. and inserting this value for all color component according to the condition in equation (2) $(8,9)$.

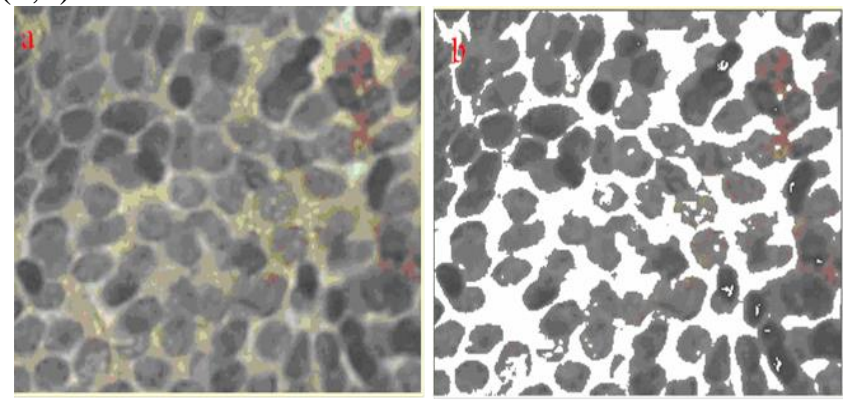

Figure (1) a-original benign image $b$ - segmented image

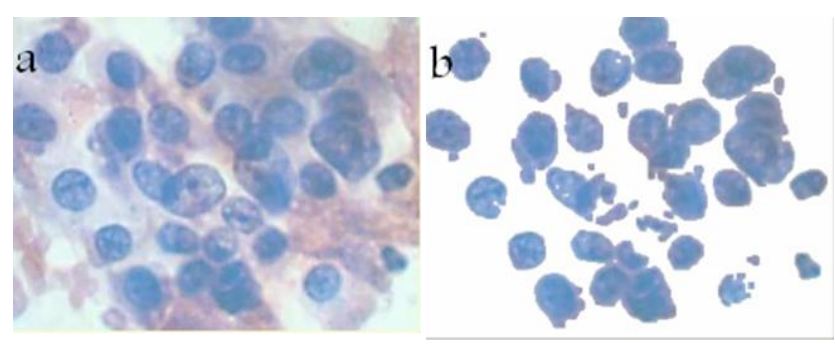

Figure (2) a-original malignant image b- segmented

\section{Conclusions}

The segmentation of cells in images -based on computing the average value of the basic (R, G, B) color component for the intercellular zones- is good and sufficient to establish other step in the study because there are many best isolated cells exist in resulted images. Three groups were considered containing the R, $\mathrm{G}$ and $\mathrm{B}$ band components of mode, median, mean, standard deviation and maximum frequency. When we input the values of mode, median, mean, std and maximum frequency in field of input in the interface of fuzzy logic program system for benign and malignant cases as see in Figure (3) for $G$ band, the result of detection appears in the right top of the program that the benign case takes the value 1 and malignant takes 2 . The accuracy obtained is equal to $97 \%$ for both $\mathrm{R}$ and $\mathrm{G}$ band while the accuracy is equal to $95 \%$.

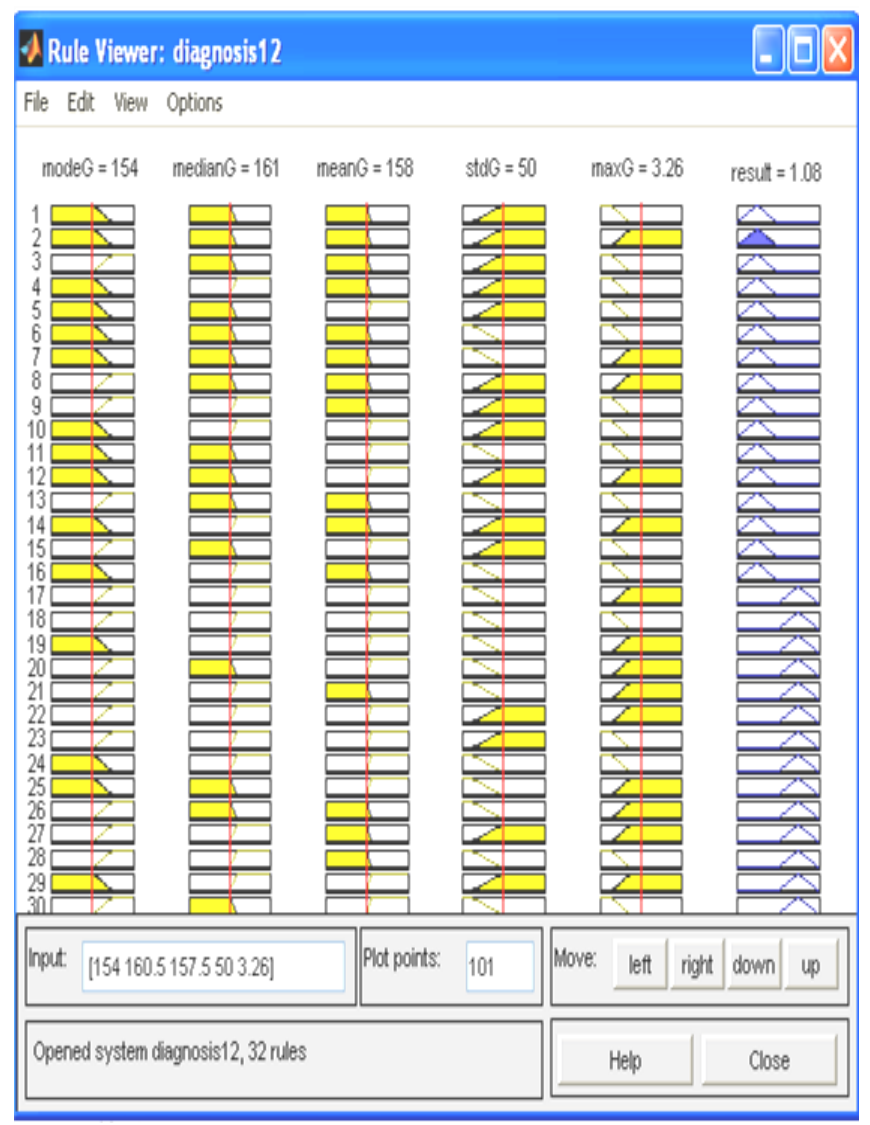

Figure (3) The interface of fuzzy logic program of statistical features of images for $\mathrm{G}$ band 


\section{References}

[1] R. C. Gonzalez, R. E. Woods and S. L. Eddins, "Digital image processing using MATLAB", January 2, (2005). http://www.amazon. com/exec/obidos/tg/detail/.

[2] Jean Philippe Thiran and Benot Macq, "Morphological feature extraction for the classification of digital images of cancerous tissues", IEEE transactions on biomedical engeneering, vol. 43, no. 10, October, pp.10111020, 1996.

[3] Isaac N. Bankman, "Handbook of medical image processing and analysis", Elsevier Inc., 2009.

[4] A. R. Katz, "Image analysis and supervised learning in the automated differentiation of white blood cells from microscopic images by a minor", M.Sc thesis, Department of Computer Science, University of RMIT, (2000).

[5] Ziad M. Abood Al-Bayati, ", "Digital Image Processing Techniques For Breast Cancer Cells Detection", Ph.D thesis, College of Education, AlMustasiriyah University, 2006.

[6] L. A. Zadeh, "On the analysis of large scale systems. In: Systems approaches and environment problems", pp. 23-27, 1974

[7] G. Chen and T. Pham, "Introduction to Fuzzy Sets, Fuzzy Logic, and Fuzzy Control Systems", CRC Press, 2001.

[8] Timothy J. Ross, "Fuzzy Logic With Engineering Application", Second Edition, John Wiley \& Sons Ltd, England, 2004.

[9] Salim J. Attia, "diagnosis of breast cancer images using fuzzy logic technique", $\mathrm{PhD}$ thesis, almustansyah university, college of science, 2012.

[10] J. M. Blackledge and D. A. Dubovitskiy, "Object Detection and Classication with Applications to Skin Cancer Screening, International Transactions on Intelligent Systems", Vol. 1, No. 1, pp. 34-45, 2008.

[11] Salim J. Attia, J. M. Blackledge, Ziad M Abood and Ibrahim $r$ agool, "diagnosis of breast cancer by optical image analysis", irish signal and system conference, Dublin,2012. 